\title{
A HIGH-YIELD PROCESS FOR THREE-DIMENSIONAL MICROELECTRODE ARRAYS
}

\author{
Qing Bai and Kensall D. Wise \\ Center for Integrated Sensors and Circuits \\ Department of Electrical Engineering and Computer Science \\ The University of Michigan \\ Ann Arbor, MI 48109-2122
}

\begin{abstract}
This paper presents a practical microassembly process for the realization of three-dimensional (3D) microelectrode arrays for recording and stimulation in the central nervous system (CNS). Several two-dimensional silicon probes are positioned in a surface platform and held parallel using micromachined spacers. Lead transfers between the probes and the platform are formed using gold beams on the probes that are attached to the platform using ultrasonic bonding with a wire-free wedge. The low-profile $(150 \mu \mathrm{m})$ outrigger design of the probes allows the bonding of fully-assembled high-density arrays. Arrays having $8 \times 16$-shanks on $200 \mu \mathrm{m}$ centers have been realized and used to record from cortical single units with interelectrode capacitances of less than lfF, corresponding to on-chip crosstalk below 0.01 percent. Micromachined tools allow full array assembly in 30 minutes.
\end{abstract}

\section{INTRODUCTION}

One- and two-dimensional micromachined silicon probes now allow the realization of passive recording and stimulating arrays having sites lithographically controlled with spacings as small as a few microns on supporting shanks $10-15 \mu \mathrm{m}$ thick and less than $30 \mu \mathrm{m}$ wide [1]. Such probes are now being used successfully by many physiologists to explore new aspects of neural systems $[2,3]$. Probes having integrated on-chip site selection, low-noise recording amplifiers, and output multiplexers/buffers have allowed the monitoring of neural activity on as many as 8 parallel channels, selected from among 32 sites, over a single signal lead $[4,5]$. Similarly, active stimulating probes have been demonstrated that are capable of generating complex current waveforms $(0$ to $\pm 127 \mu \mathrm{A} \pm 1 \mu \mathrm{A})$ on as many as 8 parallel channels and supplying them to sites selected from 64 available on 1D or 2D probes [6]. Thus, the technology is in place to record or stimulate over many parallel channels selected from a still larger number of available sites and to do so using only a few external leads. Micromachined silicon ribbon cables [7] allow chronic signal transmission between the probes and a percutaneous plug with minimal tethering.

A key remaining element needed for detailed studies of neural networks and the implementation of neural prostheses to aid the handicapped is the ability to instrument threedimensional volumes of tissue with site separations of 200$400 \mu \mathrm{m}$ while displacing no more than about $1 \%$ of the overall tissue volume. While some very novel $2 \mathrm{D}$ depth arrays have been reported [8] for cortical use, for fully 3D arrays the use of 2D silicon probes is attractive in order to take advantage of the considerable capabilities of these devices. The formation of such 3D arrays then requires the development of practical assembly techniques for submillimeter parts. Such techniques are also of interest for a variety of other sensor and micromachine applications.
In the creation of $3 D$ arrays of $2 D$ probes, the lead transfer structure between the probes and a surface platform holding the array is a major challenge. Since the probes and the platform are in perpendicular planes, the transfers must be made between orthogonal conductors. They must be robust, reliable, and formed with high-yield in a process that is reproducible in reasonable quantities and directly compatible with probe formation.

Three lead-transfer techniques have been explored. Hoogerwerf [9] employed a single subdural platform assembly, with nickel electroplated bridges between tabs on the probes and pads on the platform acting as lead transfers. Since electroplating was performed on a per-lead basis after assembly, the process was rather difficult to implement, especially for structures having large numbers of lead transfers. To allow higher throughput, an approach involving a two-platform structure (Figure 1) was next explored. In this design, each probe mounted in the subdural platform had its own ribbon cable, which was connected to a supradural platform using conventional ultrasonic bonding. Although easier to implement, the use of multiple cables complicated assembly and allowed appreciable tethering forces to be exerted on the implant as the brain moved within the cranial cavity. The number of probes and leads was also limited because of the space required for bonding at the second (supradural) platform.

This paper describes a third approach, which uses gold electroplated beam leads on the probes to form the lead transfers. These gold tabs are bent at right angles to the probes and are attached to the platform using conventional thermocompression or ultrasonic bonding. The interconnects formed are robust physically and electrically.

\section{ARRAY STRUCTURE AND FABRICATION}

The overall structure of the 3D array is shown in Figure 2. It consists of a micromachined silicon platform, a number of 2D planar probes, and two spacer bars. The probes are inserted through slots in the platform and are held orthogonal to the platform by the spacers. All of these components are fabricated on the same wafer using only a slight modification of the basic probe process. The use of outriggers on the probes for the lead transfers permits the rim of the platform to be firmly supported in a simple jig during the lead bonding. The height of the flanges (outriggers) above the platform is about $150 \mu \mathrm{m}$, which is much less than that of the central portion of the probe $(1 \mathrm{~mm})$. This minimizes the effective thickness of the rim area and allows a conventional bonding tip to fit in the $200 \mu \mathrm{m}$-wide space between the probes.

The gold beams [10] extend about $135 \mu \mathrm{m}$ below the flanges and are $100 \mu \mathrm{m}$ wide. The beams could be narrowed to $50 \mu \mathrm{m}$ to accommodate more leads if needed. They are typically electroplated to a thickness of $8-10 \mu \mathrm{m}$, can be bent easily at right angles using a custom bending tool, and are attached to pads on the platform using ultrasonic bonding. Gold beam leads are also used at the end of the cable leading from the platform to 


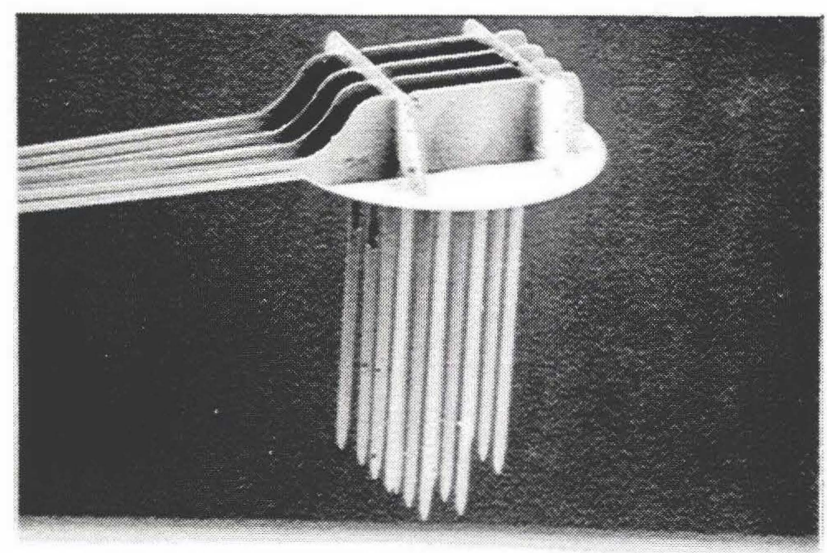

SKI $589 x$ 8245

(a)

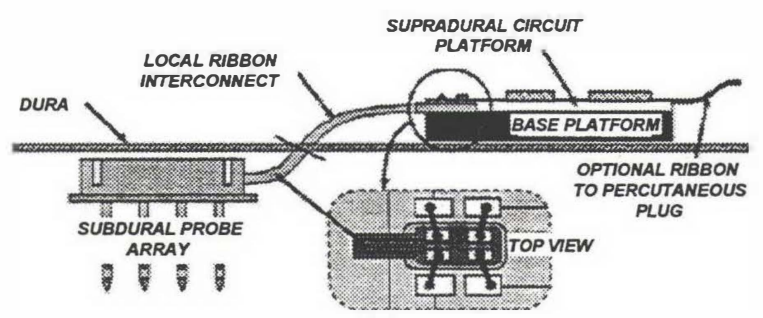

(b)

Figure 1: An approach to $3 D$ microassemblies using dedicated per-probe ribbon cables. (a) A 4x4-shank probe array implemented with this approach. (b) A schematic diagram of the approach.

a percutaneous plug. These beams extend beyond the cable to bond to an insert in the base of the plug. Conventional wire bonds are then made between pads on this insert and pins on the plug. Since the cable mounts beam upside down on the plug insert, no twisting of the cable is necessary in this assembly, minimizing any tethering force on the tissue. The gold beams are fully compatible with micromachining, form high-quality physical and electrical contacts, and allow great flexibility in creating multilayer and $3 \mathrm{D}$ microstructures.

An 8-mask fabrication sequence is used to create the 2D probe components by defining the deep boron diffusion, shallow boron diffusion, polysilicon interconnects, contacts, iridium sites, field dielectrics, site protection, and gold beams.

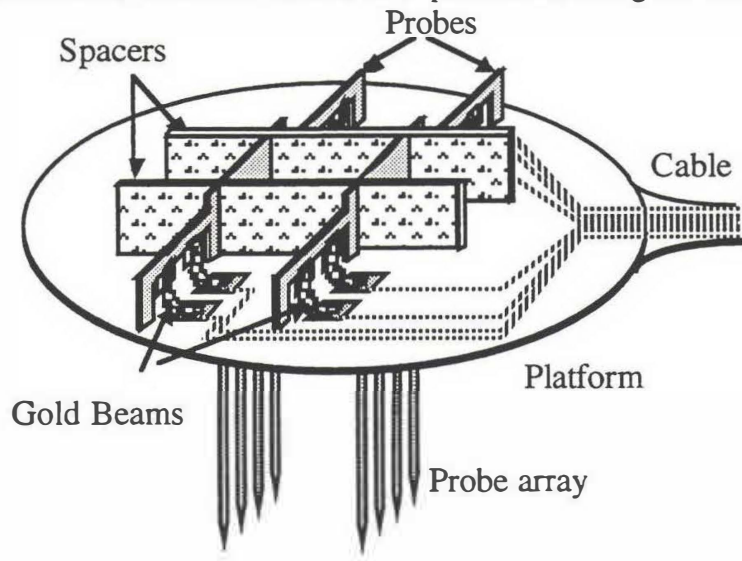

Figure 2: A high-yield approach to $3 D$ probe arrays. Lead transfers are implemented using beams that are bent at right angles and ultrasonically bonded to pads on the platform.
The fabrication process starts with a deep boron diffusion, which forms an etch-stop to define the probe, the platform, and the spacers, setting their final thicknesses at $10-15 \mu \mathrm{m}$. A shallow $(2-3 \mu \mathrm{m})$ boron diffusion is used next to form the silicon ribbon cable and the probe tips. Stress-compensated LPCVD dielectric layers $\left(300 \mathrm{~nm} / 150 \mathrm{~nm} / 300 \mathrm{~nm} \quad \mathrm{SiO}_{2} / \mathrm{Si}_{3} \mathrm{~N}_{4} / \mathrm{SiO}_{2}\right)$ are then deposited, followed by LPCVD polysilicon growth and phosphorus doping. Once the polysilicon interconnect is patterned using RIE, similar top dielectric layers are deposited. Contacts are defined using RIE, and Ti/Ir sites are then formed using sputtering and lift-off. The field dielectrics are now removed using RIE. This etch can be continued into the silicon to shape the probe sidewall if desired. A layer of photoresist is necessary to protect the Ir sites during electroplating. $\mathrm{Cr} / \mathrm{Au} / \mathrm{Cr}$ $(50 \mathrm{~nm} / 300 \mathrm{~nm} / 50 \mathrm{~nm})$ is evaporated and used as blanket conductor during Au electroplating. A thick layer of photoresist is then used to define the beams and platform pads. The top $\mathrm{Cr}$ layer is removed to expose the Au seed layer for electroplating. After the gold beams/pads are plated, the photoresist is stripped, and the $\mathrm{Au} / \mathrm{Cr}$ field layer is etched away. Finally, the wafer is thinned to approximately $100 \mu \mathrm{m}$ in a timed isotropic silicon etchant and is then given a final etch in EDP. After the components are sorted, they are ready to assemble into $3 \mathrm{D}$ probe arrays.

\section{D MICROASSEMBLY AND ASSOCIATED TOOLS}

The procedure for assembling the $3 \mathrm{D}$ arrays is carried out in four steps: bending the gold beam leads on the outriggers of the probes, assembling the probe array, bonding the beams to the pads on the platform, and connecting the probe array to the percutaneous plug. The overall process is simple and reliable, especially when facilitated with the proper assembly tools. Complete array assembly takes about 30 minutes.

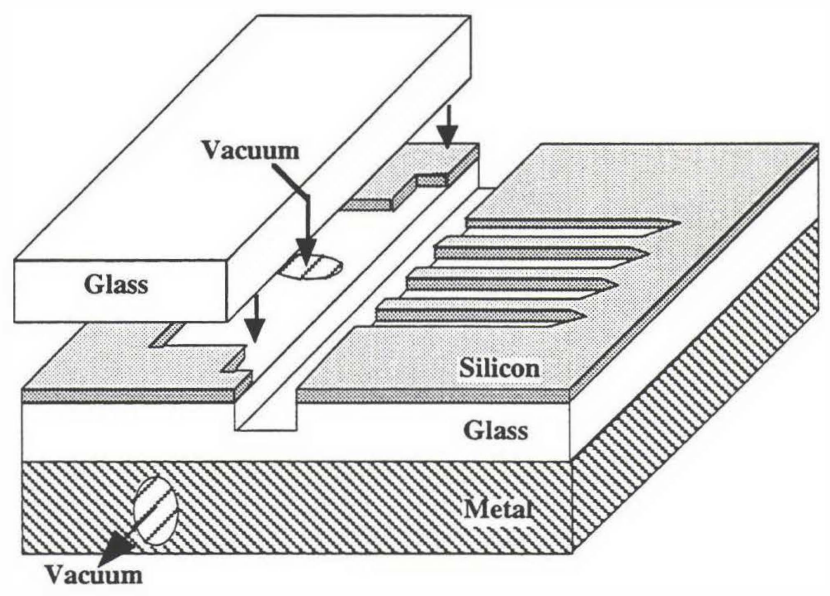

Figure 3: Perspective view of a micromachined assembly fixture used for bending the beams on probes intended for use in $3 D$ microassemblies.

It is relatively easy to bend the gold beams if the probes can be held firmly. Because the probes are very small and quite thin, ordinary clamps either do not hold them firmly enough or can break them. One way to solve this problem is to attach the probes temporarily to a glass substrate, bend the beams over the edge of the glass, and then subsequently release them. This process is simple in principle but nonetheless tedious and timeconsuming in the absence of a custom micromachined assembly tool. 


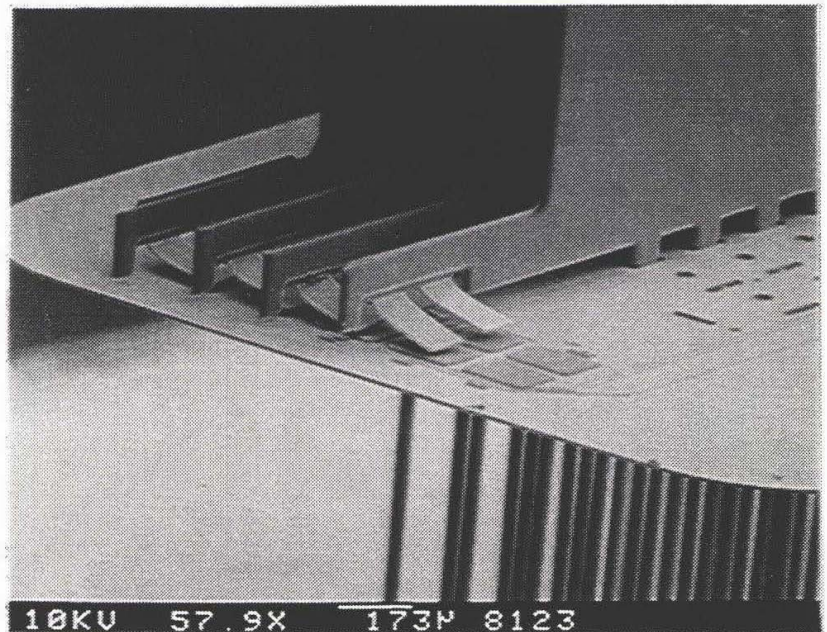

Figure 4: A partially-assembled probe array, showing the beam lead transfers before bonding.

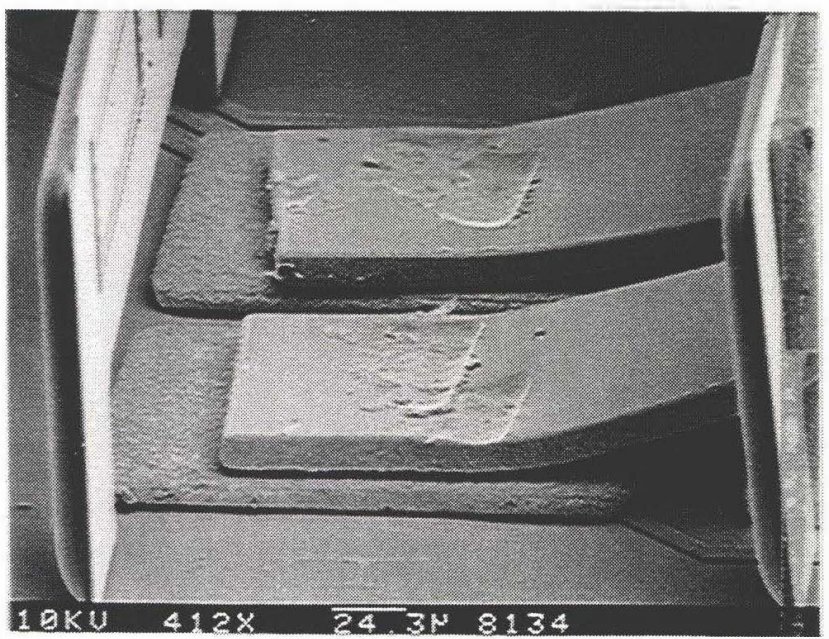

Figure 5: View of two ultrasonically-bonded lead transfers.

Figure 3 shows the schematic diagram of such a tool. A mold is lithographically defined to match the shape of the probes being used. A vacuum is applied to the mold fixture to hold the probe in it, and a piece of glass is used to clamp the probe firmly in position. Only one mask is needed for the formation of such a fixture. A deep boron diffusion is first used to define the mold recess. After drilling a vacuum access hole in a glass substrate, the glass is anodically bonded to the silicon mold wafer, and the wafer is then etched in EDP to remove all but the boron-doped areas. Thus, a probe mold is formed on the glass substrate. A groove $200 \mu \mathrm{m}$ wide and $200 \mu \mathrm{m}$ deep is next formed using a wafer saw. Such saw cuts produce a very clean edge. The mold is then glued to a metal support base having a corresponding vacuum hole to complete the fixture.

With this tool, the beams can be easily bent over into the groove in the glass using a special bending tool. The beams can be bent in either direction, forward or backward, and we have chosen to bend the beams backward under the probe so that direct gold-to-gold bonds can be made to the platform pads. A short overhang of dielectric under the beam prevents shorts to the probe substrate.

With the beams bent, the probes are inserted into slots in the platform, which is held on the mounting stage of a dedicated microassembly station equipped with two three-axis micromanipulators. Individual holes are used for each shank in order to give the center of the platform as much strength as possible. In addition, holes have been placed in the platform between probes to encourage tissue to grow through the platform to anchor it to the cortical surface.

It was initially very difficult to put the spacers in place when mounting the probes. The probes are not initially parallel to each other and tend to stick back to back when the spacer is put in. We therefore use a micromachined comb structure on a second manipulator to separate the probes one by one from the side. With the spacers in place, the fork is removed. The fork structure has $50 \mu \mathrm{m}$ spaces between the tines of the comb, with shanks that are tapered from one side to the other. It is very easy to use and replace. Figure 4 shows a partially assembled array of four probes before bonding.

Once the probe array is cemented together using a drop of Silastic, the beams are bonded to pads on the platform using conventional ultrasonic bonding with a wire-free wedge, as shown in Figure 5. Finally, the bonding areas are covered with Silastic to provide good insulation over the lead transfers. Figure 5 shows two views of a fully-assembled 3D array of eight probes and 128 shanks (an $8 \times 16$-shank array). The probe-toprobe separation is $200 \mu \mathrm{m}$, which matches the shank-to-shank distance. The maximum shank length is $2.5 \mathrm{~mm}$. The height of the central part of the probe above the platform $(1 \mathrm{~mm})$ mimics what will be required for CMOS multiplexing circuitry on active arrays using $3 \mu \mathrm{m}$ features.

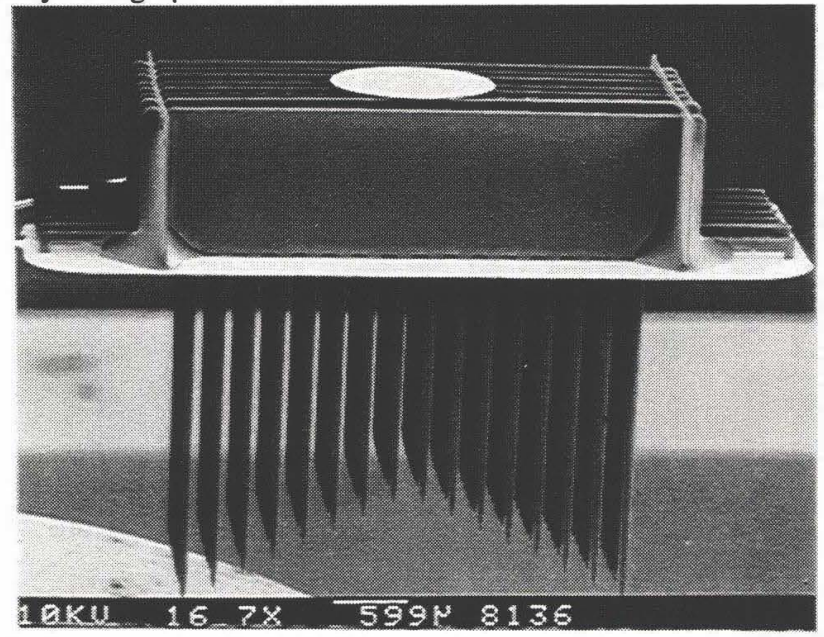

(a)

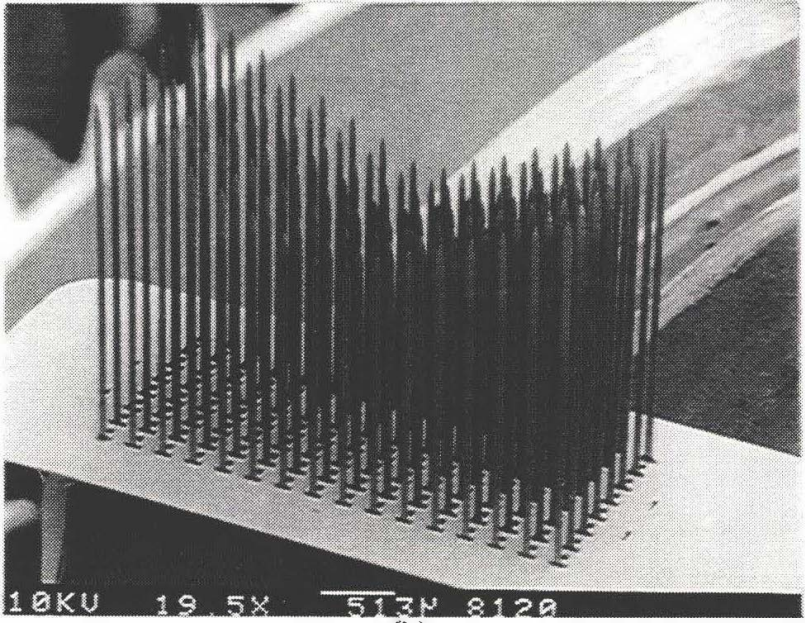

(b)

Figure 6: SEM views of an 8x16-shank 8-probe array. The shanks are on $200 \mu m$ centers. (a) Top view. (b) Bottom view. 


\section{EXPERIMENTAL RESULTS}

For testing purposes, each of the probes in these arrays has four sites that are brought out to pads on the platform. The leads from the center three probes connect to a 12-pin percutaneous plug via an integrated ribbon cable. These arrays have been successfully tested in-vitro and in-vivo. The typical site impedance at $1 \mathrm{kHz}$ is about $2 \mathrm{M} \Omega$ before iridium activation, which represents worst case from the standpoint of signal transmission. An equivalent circuit of the probe-platform-cable assembly is shown in Figure 7. Without on-chip circuitry, the signal attenuation is less than $10 \%$ (set by the shunt capacitance of $18 \mathrm{pF}$ ), with interchannel crosstalk (set by the connector and external leads) below $0.5 \%$. Since the interchannel coupling on the assembly itself is less than $1 \mathrm{fF}$, with on-chip signal amplification and buffering the crosstalk should be $<0.01 \%$, making simultaneous recording and stimulation possible. Typical neural recordings in guinea pig cortex are shown in Figure 8. A series of chronic implants are now being undertaken to assess the tissue reaction to such arrays.

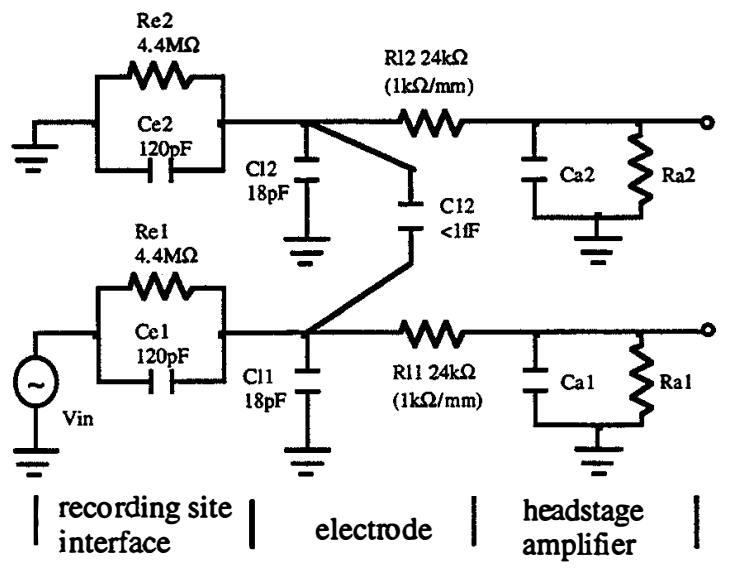

Figure 7: An equivalent circuit showing the various electrical elements that form the signal path in a typical probe assembly.

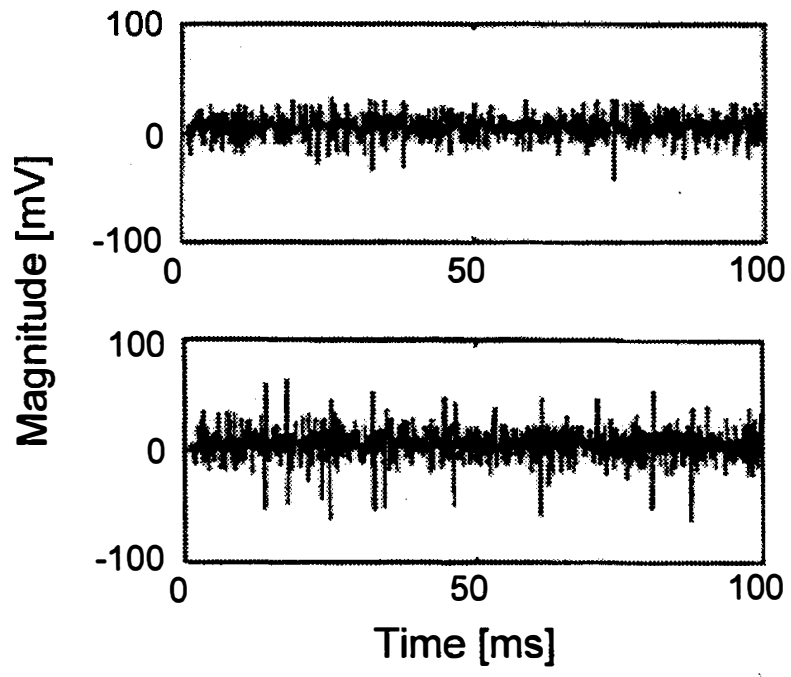

Figure 8: Simultaneous single-unit activity recorded from guinea pig cortex using an $8 \times 8$-shank $3 D$ probe array.

\section{CONCLUSIONS}

Gold electroplated beams are being used to realize orthogonal lead ransfers in forming 3D microelectrode arrays. This approach, implemented using simple micromachined assembly tools and fixtures, meets the needs of reliable array fabrication with high yield. It is easier to implement than previous approaches, is more reliable in terms of its interconnections, consumes less bonding area, and results in less tethering force on the tissue. The entire assembly procedure for an 8x16-shank array can be finished within half an hour. In further extending this approach to large numbers of sites, however, active multiplexing on the probes will be required. Using active multiplexing, arrays having 1024 sites on $200-400 \mu \mathrm{m}$ centers over $8-64 \mathrm{~mm}^{3}$ issue volumes are being developed.

\section{ACKNOWLEDGMENTS}

The authors wish to thank Drs. F. T. Hambrecht and W. J. Heetderks of the Neural Prosthesis Program, National Institutes of Health (NINDS), for their guidance and support of this work under contract NIH-NINDS-NO1-NS-4-2303.

\section{REFERENCE}

1. K. Najafi, K. D. Wise, and T. Mochizuki, "A High-Yield ICCompatible Multichannel Recording Array," IEEE Trans. Electron Devices, 32, pp. 1206-1211, July 1985.

2. A. Bragin, G. Jando, Z. Nadasdy, J. Hetke, K. Wise, and G. Buzsaki, "Gamma $(40 \mathrm{~Hz}-100 \mathrm{~Hz})$ Oscillation in the Hippocampus of the Behaving Rat," J. Neuroscience, 15, pp. 47-60, January 1995.

3. R. R. Carter and J. C. Houk, "Multiple Single-Unit Recordings from the CNS using Thin-Film Electrode Arrays," IEEE Trans. Rehab. Engr., 1, pp. 175-184, September 1993.

4. J. Ji and K. D. Wise, "An Implantable CMOS Circuit Interface for Multiplexed Microelectrode Recording Arrays," IEEE J. Solid-State Circuits, 27, pp. 433-443, March 1992.

5. J. L. Lund and K. D. Wise, "Chip-Level Encapsulation of Implantable CMOS Microelectrode Arrays," Digest Solid-State Sensor and Actuator Workshop, Hilton Head, S.C., pp. 29-32, June 1994.

6. C. Kim and K. D. Wise, "A 64-Site Multiplexed Low-Profile Neural Probe with On-Chip CMOS Circuitry," Digest 1994 IEEE Symposium on VLSI Circuits, Honolulu, June 1994.

7. J. F. Hetke, J. L. Lund, K. Najafi, K. D. Wise, and D. J. Anderson, "Silicon Ribbon Cables for Chronically-Implantable Microelectrode Arrays," IEEE Trans. Biomed. Engr., 41, pp. 314-321, April 1994.

8. P. K. Campbell, K. E. Jones, R. J. Huber, K. W. Horch, and R. A. Normann, "A Silicon-Based Three-Dimensional Neural Interface: Manufacturing Processes for an Intracortical Electrode Array," IEEE Trans. Biomed. Engr., 38, pp. 758-767, August 1991.

9. A. C. Hoogerwerf and K. D. Wise, "A Three-Dimensional Microelectrode Array for Chronic Neural Recording," IEEE Trans. Biomed. Engr., 41, pp. 1136-1146, December 1994.

10. M. P. Lepselter, "Beam-Lead Technology", Bell System Technical Journal, 45, pp. 233-254, February 1966. 